Research Article

\title{
Continuous Integral Terminal Sliding Mode Control for Double-Layer Peltier System Based on Finite-Time Observer
}

\author{
Congyan Chen (D), Wenbin Song, and Shichen Ding \\ School of Automation, Southeast University, Nanjing, China \\ Correspondence should be addressed to Congyan Chen; chency@seu.edu.cn
}

Received 5 February 2020; Revised 4 April 2020; Accepted 13 April 2020; Published 5 May 2020

Academic Editor: Alessandro Lo Schiavo

Copyright ( $) 2020$ Congyan Chen et al. This is an open access article distributed under the Creative Commons Attribution License, which permits unrestricted use, distribution, and reproduction in any medium, provided the original work is properly cited.

\begin{abstract}
This paper addresses the finite-time tracking problem for the double-layer Peltier system. Peltier is a semiconductor thermoelectrical transform device. It is widely used in the thermal tactile reappearance area. To expand temperature differences, Peltier is usually used in the form of double layers. There are some uncertain factors such as state coupling, external disturbance, and parameter perturbation in double-layer Peltier. Therefore, it is of great theoretical and practical significance to design a controller with superior performance. To this end, a compound continuous integral terminal sliding mode control strategy is proposed here. Firstly, finite-time disturbance observers are designed for feedforward compensation and evaluating the external disturbances. Secondly, the strong robustness of the sliding mode control enhances the disturbance rejection of the system. The continuous integral sliding mode makes the input continuous and weakens the chattering. Also, the terminal sliding mode improves the convergence speed of the system on the sliding surface significantly. The performance of the proposed method is analyzed through Lyapunov stability analysis, simulations, and experiments. Compared to nonsmooth finite-time control, the continuous integral terminal sliding mode control achieves rapid temperature stability and better disturbance rejection under the same condition of finite-time convergence.
\end{abstract}

\section{Introduction}

Single-layer Peltier achieves thermal tactile reappearance within a certain range [1]. In order to expand application, it is necessary to enlarge temperature differences. Actually, with the saturation of the control input, single-layer Peltier cannot achieve a satisfactory temperature effect. In addition, single-layer Peltier has limited refrigeration capacity, which cannot meet the demand of thermal tactile reappearance in low-temperature environment [2]. Considering that multiple Peltiers can be combined for refrigeration, a doublelayer Peltier temperature control system is adopted in this paper. Two Peltier chips are placed up and down to form a double-layer Peltier device. The top Peltier and the bottom Peltier work, respectively, under their control current. The double-layer Peltier device is probably used in a lower temperature environment, which makes the thermal tactile reappearance with a larger temperature difference [3].

However, the control system nonlinearity becomes stronger with the number of the layers increased [4]. Also, the temperature control algorithm with satisfactory performance is also needed to make sure that the thermal tactile perception reappearance device based on a Peltier chip can reproduce the temperature timely and accurately [5]. The convergence speed of the common control algorithm is relatively slow. Even if the response of the Peltier chip is fast, it cannot realize thermal tactile reappearance timely. Similarly, the disturbance rejection performance of the traditional control algorithms is not strong, so the device itself cannot effectively overcome the thermal flow disturbance of fingers. Then, the accurate thermal tactile reappearance will be impossible. Therefore, convergence and disturbance rejection are two important indicators to measure the performance of the thermal tactile reappearance system, and it is very important to design a temperature control algorithm with fast convergence speed and strong robustness.

In our previous study [6] on double-layer Peltier, based on the feedforward compensation of disturbance, a nonsmooth finite-time feedback control strategy was adopted to ensure that the state can converge to the equilibrium point in 
finite time. Under the action of fractional power, the nonsmooth control enables the controller to have larger control input near the equilibrium point, so that the system can achieve a faster convergence performance [7]. However, this method is based on the premise that the disturbance can be precisely compensated. When the disturbance estimation is inaccurate or there still exist unknown disturbances without compensation, the controller cannot perform perfectly and accurately. Since the sliding mode control has a strong robustness $[8,9]$, the sliding mode control can exactly make up for this defect which can be suppressed by the gain of sliding mode control and the switching characteristic of sliding mode control. Therefore, sliding mode control is also widely used in electromechanical systems [10-12].

However, sliding mode control inevitably has problems about chattering [13], relative order limitation, and control precision [14]. The fundamental cause of chattering problems is the discontinuity of the control input. In order to eliminate chattering problems and obtain continuous control, a continuous sliding mode is adopted $[15,16]$. Continuous sliding mode adds the discontinuous switching signal to the derivative of the control signal. After the process of filtering, the actual control input does not contain high-frequency switching signal, and the control input becomes continuous. Therefore, the chattering is essentially reduced. In addition, for the conventional sliding mode control, the order of the sliding mode surface is one order lower than that of the system. After modeling, it is found that Peltier is a first-order system. Conventionally designing the sliding mode surface will lose its dynamic characteristics and result in considerable fluctuations in its input. In this paper, the order of the sliding mode surface is not reduced, so that the same dynamic model as the system order is adopted by the integral sliding mode control [17]. Integral sliding mode control can reduce the steady-state error, enhance the stability of the controller, and reduce chattering to a certain extent [18]. In order to ensure the finite-time convergence of the double-layer Peltier temperature control system, the disturbance observer with finite-time convergence is also adopted. For traditional integral sliding mode control, a linear sliding mode surface is used. In order to improve the convergence speed of the tracking error, the terminal sliding mode control strategy is used widely [19-21]. The terminal sliding mode control strategy is to use uses a nonlinear terminal sliding mode surface instead of a linear sliding mode surface. The biggest advantage of terminal sliding mode control is its fast convergence speed. In addition, it has small steady-state error and high control accuracy. Meanwhile, the terminal sliding mode surface is adopted in the selection of the sliding mode surface [22].

The structure of this paper is organized as follows. Firstly, Section 2 introduces the modeling of the temperature control system. Secondly, Section 3 introduces the design of the compound sliding mode control strategy based on the system model. In Section 4, some simulation and experiment results are given to verify the effectiveness and superiority of the algorithm. Last but not least, the conclusion of this paper is given in Section 5 .

\section{Modeling of Temperature Control System Based on Double-Layer Peltier}

The schematic diagram of the thermal tactile reproduction device based on double-layer Peltier is shown in Figure 1. Double-layer Peltier superimposes two Peltier chips together. When the finger with higher temperature $\left(T_{f}\right)$ contacts the top Peltier surface, the thermal flow $\left(q_{\text {dis }}\right)$ is generated. In order to maintain a constant temperature $\left(T_{h}\right)$, the top Peltier is affected by the input current and then creates heat transfer $\left(q_{p 1}\right)$ from top to bottom because of the Peltier effect. Also, the bottom-layer Peltier generates topdown heat transfer $\left(q_{p 2}\right)$ under the action of input current in the bottom layer. The upper surface of Peltier is the cold end, while the lower surface is the hot end. At the hot end of the bottom Peltier, there is a heat sink to dissipate heat and maintain a constant temperature approximately. Cold ends are equipped with temperature sensors to detect the surface temperatures $\left(T_{c 1}\right.$ and $\left.T_{c 2}\right)$, as shown in Figure 1.

According to the single-layer Peltier equivalent circuit diagram [23] and reference [6], the equivalent circuit diagram can be obtained, as shown in Figure 2.

From Figure 2, the following equation can be obtained referring to Kirchhoff's law in the circuit:

$$
\left\{\begin{array}{l}
C_{p 1} \frac{\mathrm{d} T_{c 1}}{\mathrm{~d} t}=\frac{1}{R_{p 1}}\left(T_{c 2}-T_{c 1}\right)-\alpha T_{c 1} I_{1}+\frac{1}{2} R_{e l} I_{1}^{2}+q_{\mathrm{dis}} \\
C_{p 2} \frac{\mathrm{d} T_{c 2}}{\mathrm{~d} t}=\frac{1}{R_{p 1}}\left(T_{c 1}-T_{c 2}\right)-\alpha T_{c 2} I_{2}+\frac{1}{2} R_{e l} I_{2}^{2}+\frac{1}{R_{p 2}} T_{m}
\end{array}\right.
$$

where $T_{m}=T_{h}-T_{c 2}$. Defining the state variables $x_{1}=T_{c 1}$ and $x_{2}=T_{c 2}$ and inputs $u_{1}=I_{1}$ and $u_{2}=I_{2}$, then (1) can be rewritten as

$$
\left\{\begin{array}{l}
\dot{x}_{1}=a_{1} x_{1}+b_{1} x_{1} u_{1}+\psi_{1} x_{2}+c_{1} u_{1}^{2}+d_{1}, \\
\dot{x}_{2}=a_{2} x_{2}+b_{2} x_{2} u_{2}+\psi_{2} x_{1}+c_{2} u_{2}^{2}+d_{2},
\end{array}\right.
$$

where $a_{1}=-\left(1 /\left(C_{p 1} R_{p 1}\right)\right), b_{1}=-\left(\alpha / C_{p 1}\right), \psi_{1}=1 /\left(C_{p 1} R_{p 1}\right)$, $c_{1}=R_{e l} / 2 C_{p 1}, \quad d_{1}=\left(q_{\text {dis }} / C_{p 1}\right), \quad a_{2}=-\left(1 /\left(C_{p 2} R_{p 1}\right)\right)-$ $\left(1 /\left(C_{p 2} R_{p 2}\right)\right), \quad b_{2}=-\left(\alpha / C_{p 2}\right), \quad \psi_{2}=1 /\left(C_{p 2} R_{p 1}\right), \quad$ and $c_{2}=R_{e l} / 2 C_{p 2}, d_{2}=T_{h} /\left(C_{p 2} R_{p 2}\right)$.

\section{Continuous Integral Terminal Sliding Mode Control Based on Disturbance Observer}

In order to improve the control performance of the system, the terminal sliding mode controller [24] based on a finitetime observer is designed in this section. Firstly, a finite-time disturbance observer [25] is designed to estimate the disturbance of the system. Then, an integral terminal sliding mode surface with the same order as system (2) is 


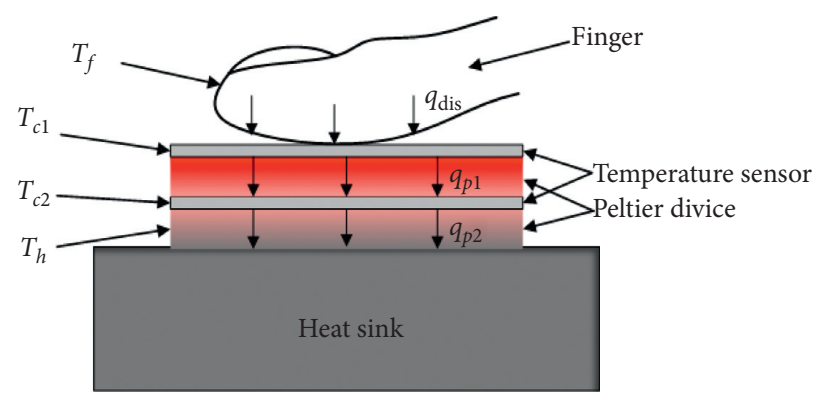

FIgURE 1: Schematic diagram of the thermal tactile reappearance device based on double-layer Peltier.

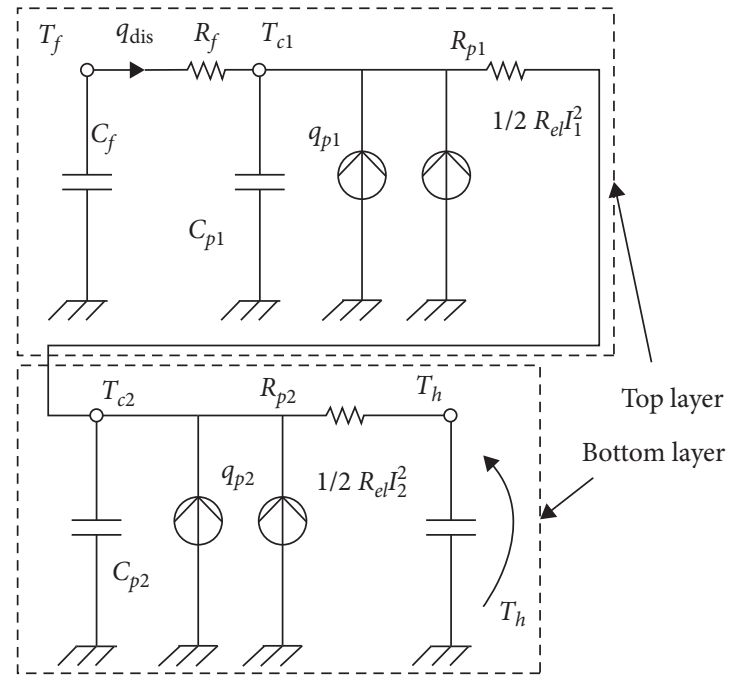

FIGURE 2: Equivalent circuit diagram of the thermal tactile reappearance device based on double-layer Peltier.

constructed to maintain the dynamic characteristics of the sliding mode surface. Finally, in order to eliminate chattering and ensure the continuity of the control input, a discontinuous control signal is added to the first derivative of the switching control input by the continuous sliding mode [26].

Assumption 1: it is assumed that the disturbances $d_{1}$ and $d_{2}$ of system (2) are bounded and have first derivatives, and their derivatives $\dot{d}_{1}$ and $\dot{d}_{2}$ are bounded by positive constants $K_{1}$ and $K_{2}$, respectively.

To estimate the disturbances $d_{1}$ and $d_{2}$ in system (2), two finite-time disturbance observers are designed as follows:

$$
\left\{\begin{array}{l}
\dot{\hat{x}}_{1}=a_{1} x_{1}+b_{1} x_{1} u_{1}+c_{1} u_{1}^{2}+\psi_{1} x_{2}+\widehat{z}_{1}+k_{11} \operatorname{sig}^{m_{21}}\left(x_{1}-\hat{x}_{1}\right), \\
\dot{\bar{z}}_{1}=\dot{\vec{d}}_{1}=\widehat{z}_{2}+k_{21} \operatorname{sig}^{m_{31}}\left(x_{1}-\widehat{x}_{1}\right), \\
\dot{\bar{z}}_{2}=k_{31} \operatorname{sig}^{m_{41}}\left(x_{1}-\widehat{x}_{1}\right),
\end{array}\right.
$$

$$
\left\{\begin{array}{l}
\dot{\vec{x}}_{2}=a_{2} x_{2}+b_{2} x_{2} u_{2}+c_{2} u_{2}^{2}+\psi_{2} x_{1}+\widehat{z}_{3}+k_{12} \operatorname{sig}^{m_{22}}\left(x_{2}-\widehat{x}_{2}\right), \\
\dot{\vec{z}}_{3}=\dot{\hat{d}}_{2}=\widehat{z}_{4}+k_{22} \operatorname{sig}^{m_{32}}\left(x_{2}-\widehat{x}_{2}\right), \\
\dot{\vec{z}}_{4}=k_{32} \operatorname{sig}^{m_{42}}\left(x_{2}-\widehat{x}_{2}\right)
\end{array}\right.
$$

where $\operatorname{sig}^{\alpha}(x)=\operatorname{sign}(x)|x|^{\alpha}, \quad m_{i 1}=1+(i-1) \tau, \quad m_{i 2}=1+$ $(i-1) \tau, i=1,2,3,4, \tau \in(-1 / 3,0), \hat{x}_{1}$ and $\hat{x}_{2}$ are the estimations of system states $x_{1}$ and $x_{2}$, respectively, $z_{1}=d_{1}$, $z_{2}=\dot{d}_{1}, z_{3}=d_{2}, z_{4}=\dot{d}_{2}, \widehat{z}_{1}$, and $\widehat{z}_{2}$ are observations of $d_{1}$ and its derivatives, and $\widehat{z}_{3}$ and $\widehat{z}_{4}$ are observations of $d_{2}$ and its derivatives.

Taking $x_{1 \text { ref }}$ and $x_{2 \text { ref }}$ as the reference temperature values of $x_{1}$ and $x_{2}$, tracking errors are defined as $e_{1}=x_{1}-$ $x_{1 \text { ref }}$ and $e_{2}=x_{2}-x_{2 \text { ref }}$, disturbance observation error states as $\widetilde{d}_{1}=\widehat{d}_{1}-d_{1}$ and $\widetilde{d}_{2}=\widehat{d}_{2}-d_{2}$, and disturbance derivative observation error states as $\dot{\vec{d}}_{1}=\hat{d}_{1}-\dot{d}_{1}$ and $\tilde{d}_{2}=\widehat{d}_{2}-\dot{d}_{2}$.

In finite-time disturbance observers (3) and (4), referring to the proof of 3.3.1 in reference [25], for any $\tau \in(-1 / 3,0)$, there exist $k_{11}, k_{21}, k_{31}$, and finite time $t_{f 1}$ to make the observation error states satisfy $\left|x_{1}-\widehat{x}_{1}\right| \leq \delta_{11},\left|d_{1}-\widehat{d}_{1}\right| \leq \delta_{21}$, and $\left|\dot{d}_{1}-\dot{\vec{d}}_{1}\right| \leq \delta_{31}$ when $t_{1} \leq t_{f 1}$, where $\delta_{11}, \delta_{21}$, and $\delta_{31}$ are constants. Similarly, there also exists $k_{12}, k_{22}, k_{32}$, and finite time $t_{f 2}$ to make the observation error. states satisfy $\left|x_{2}-\widehat{x}_{2}\right| \leq \delta_{12}, \quad\left|d_{2}-\widehat{d}_{2}\right| \leq \delta_{22}$, and $\left|\dot{d}_{2}-\dot{\vec{d}}_{2}\right| \leq \delta_{32}$ when $t_{2} \leq t_{f 2}$, where $\delta_{12}, \delta_{22}$, and $\delta_{32}$ are constants.

In the double-layer Peltier system (2), $x_{1}$ and $x_{2}$ can be obtained by temperature sensors. In order to eliminate the coupling of input states, two auxiliary states are designed as follows: 


$$
\left\{\begin{array}{l}
v_{1}=v_{c 1}+v_{s w 1}=b_{1} x_{1} u_{1}+c_{1} u_{1}^{2} \\
v_{2}=v_{c 2}+v_{s w 2}=b_{2} x_{2} u_{2}+c_{2} u_{2}^{2}
\end{array}\right.
$$

where $v_{c i}$ and $v_{s w i}(i=1,2)$ represent the control input and the switching control input in the Peltier layer.

The state error of the system can be rewritten as

$$
\left\{\begin{array}{l}
\dot{e}_{1}=a_{1} x_{1}+\psi_{1} x_{2}+v_{1}+d_{1}, \\
\dot{e}_{2}=a_{2} x_{2}+\psi_{2} x_{1}+v_{2}+d_{2} .
\end{array}\right.
$$

In the top and bottom Peltier control system, the firstorder terminal sliding mode surface is designed as follows:

$$
\left\{\begin{array}{l}
s_{1}=\dot{e}_{1}+\eta_{1} \operatorname{sign}\left(e_{1}\right)\left|e_{1}\right|^{\gamma_{1}}, \\
s_{2}=\dot{e}_{2}+\eta_{2} \operatorname{sign}\left(e_{2}\right)\left|e_{2}\right|^{\gamma_{2}},
\end{array}\right.
$$

where $\eta_{1}, \eta_{2}>0$ and $0<\gamma_{1}, \gamma_{2}<1$.

The control inputs are designed as

$$
\left\{\begin{array}{l}
v_{c 1}=-\eta_{1} \operatorname{sign}\left(e_{1}\right)\left|e_{1}\right|^{\gamma_{1}}-a_{1} x_{1}-\psi_{1} x_{2}-\widehat{d}_{1}, \\
v_{c 2}=-\eta_{2} \operatorname{sign}\left(e_{2}\right)\left|e_{2}\right|^{\gamma_{2}}-a_{2} x_{2}-\psi_{2} x_{1}-\widehat{d}_{2},
\end{array}\right.
$$

where $\widehat{d}_{1}$ and $\hat{d}_{2}$ are the estimations of disturbances $d_{1}$ and $d_{2}$, respectively, which can be obtained from the observers (3) and (4).

The derivative of switching control inputs is designed as

$$
\left\{\begin{array}{l}
\dot{v}_{s w 1}=-k_{1} \operatorname{sign}\left(s_{1}\right), \\
\dot{v}_{s w 2}=-k_{2} \operatorname{sign}\left(s_{2}\right),
\end{array}\right.
$$

where $k_{1}, k_{2}>0$.

Theorem. Under assumption 1, for the double-layer Peltier system (2), if the finite-time disturbance observers are designed as (3) and (4), the sliding mode surfaces are chosen as (9) and the control strategy is designed as follows:

$$
\left\{\begin{array}{l}
u_{1}=-\frac{b_{1} x_{1}}{2 c_{1}}+\frac{1}{2 c_{1}} \sqrt{b_{1}^{2} x_{1}^{2}+4 c_{1}\left(v_{c 1}+v_{s w 1}\right)}, \\
u_{2}=-\frac{b_{2} x_{2}}{2 c_{2}}+\frac{1}{2 c_{2}} \sqrt{b_{2}^{2} x_{2}^{2}+4 c_{2}\left(v_{c 2}+v_{s w 2}\right)},
\end{array}\right.
$$

then the systems states $x_{1}$ and $x_{2}$ can reach their desired values $x_{1 \text { ref }}$ and $x_{2 \text { ref }}$ in finite time.

Proof. Substituting equations (5) and (8) into the state-error system (6) to get the closed-loop error system

$$
\begin{aligned}
\dot{e}_{1}= & a_{1} x_{1}+\psi_{1} x_{2}+v_{c 1}+v_{s w 1}+d_{1} \\
= & a_{1} x_{1}+\psi_{1} x_{2}-\eta_{1} \operatorname{sign}\left(e_{1}\right)\left|e_{1}\right|^{\gamma_{1}}-a_{1} x_{1}-\psi_{1} x_{2} \\
& -\widehat{d}_{1}+v_{s w 1}+d_{1} \\
= & v_{s w 1}-\eta_{1} \operatorname{sign}\left(e_{1}\right)\left|e_{1}\right|^{\gamma_{1}}-\tilde{d}_{1},
\end{aligned}
$$

we have

$$
\dot{e}_{2}=v_{s w 2}-\eta_{2} \operatorname{sign}\left(e_{2}\right)\left|e_{2}\right|^{\gamma_{2}}-\tilde{d}_{2} \text {. }
$$

Substituting equations (11) and (12) into equation (7), it obtains

$$
\left\{\begin{array}{l}
s_{1}=v_{s w 1}-\tilde{d}_{1}, \\
s_{2}=v_{s w 2}-\tilde{d}_{2} .
\end{array}\right.
$$

Lemma. Assuming that continuously differentiable function $V: U \longrightarrow R$ satisfies the following conditions:

(i) $V$ is a positive definite function,

(ii) $\dot{V}(x)+c V^{\alpha}(x) \leq 0, x \in U_{0} \backslash\{0\}$, where $c>0$, $\alpha \in(0,1)$ and $U_{0} \subset U$.

When $U=U_{0}=R^{n}$, the system is stable in finite time [27].

The stability analysis of the error dynamics (11) and (12) are given by the following three steps:

(1) Proving that the sliding mode surface and the system state error are bounded in finite time.

Selecting boundary function $V_{A}=(1 / 2) s_{1}^{2}+(1 / 2) e_{1}^{2}+$ $(1 / 2) s_{2}^{2}+(1 / 2) e_{2}^{2}$, the derivation of $V_{A}$ can be obtained as follows:

$$
\begin{aligned}
\dot{V}_{A}= & e_{1} \dot{e}_{1}+e_{2} \dot{e}_{2}+s_{1} \dot{s}_{1}+s_{2} \dot{s}_{2} \\
= & e_{1}\left(s_{1}-\eta_{1} \operatorname{sign}\left(e_{1}\right)\left|e_{1}\right|^{\gamma_{1}}\right)+s_{1}\left(\dot{v}_{s w 1}-\dot{\tilde{d}}_{1}\right) \\
& +e_{2}\left(s_{2}-\eta_{2} \operatorname{sign}\left(e_{2}\right)\left|e_{2}\right|^{\gamma_{2}}\right)+s_{2}\left(\dot{v}_{s w 2}-\dot{\tilde{d}}_{2}\right) \\
= & e_{1} s_{1}-\eta_{1}\left|e_{1}\right|^{\gamma_{1}+1}+e_{2} s_{2}-\eta_{2}\left|e_{2}\right|^{\gamma_{2}+1}-k_{1}\left|s_{1}\right| \\
& -s_{1} \dot{\tilde{d}}_{1}-k_{2}\left|s_{2}\right|-s_{2} \dot{\tilde{d}}_{2} \\
\leq & \frac{1}{2} e_{1}^{2}+\frac{1}{2} e_{2}^{2}+s_{1}^{2}+s_{2}^{2}+\frac{1}{2} \dot{\vec{d}}_{1}^{2}+\frac{1}{2} \dot{\vec{d}}_{2}^{2} \\
\leq & 2 V_{A}+\frac{1}{2}\left|\dot{\vec{d}}_{1 \max }\right|^{2}+\frac{1}{2}\left|\dot{\vec{d}}_{2 \max }\right|^{2},
\end{aligned}
$$

where $\left|\dot{\tilde{d}}_{1 \max }\right|$ and $\left|\dot{\vec{d}}_{2 \max }\right|$ are the upper bounds of $\left|\tilde{d}_{1}\right|$ and $\left|\tilde{d}_{2}\right|$. According to inequality (14), $V_{A}$ is bounded in finite time. Therefore, $s_{1}, s_{2}, e_{1}$, and $e_{2}$ cannot escape to infinity in finite time [28].

(2) Proving that the top and bottom Peltier control systems reach to their respective sliding mode surface in finite time.

Choosing Lyapunov function $V_{1}=(1 / 2) s_{1}^{2}$ for the top Peltier control system, then

$$
\begin{aligned}
\dot{V}_{1} & =s_{1} \dot{s}_{1} \\
& =s_{1}\left(\dot{v}_{s w 1}-\dot{\widetilde{d}}_{1}\right) \\
& =s_{1}\left(-k_{1} \operatorname{sign}\left(s_{1}\right)-\dot{\tilde{d}}_{1}\right) \\
& =-k_{1}\left|s_{1}\right|-s_{1} \dot{\tilde{d}}_{1} .
\end{aligned}
$$

Similarly, in the bottom Peltier control system, choose Lyapunov function $V_{2}=(1 / 2) s_{2}^{2}$, then 
$\dot{V}_{2}=-k_{2}\left|s_{2}\right|-s_{2} \dot{\tilde{d}}_{2}$. According to reference [25], $\dot{\tilde{d}}_{1}$ and $\dot{\vec{d}}_{2}$ can be bounded by positive constants $\delta_{31}$ and $\delta_{32}$, respectively. Therefore, if $k_{1}$ is selected such that $k_{1} \geq \delta_{31}$, then we have $\dot{V}_{1} \leq-\left(k_{1}-\delta_{31}\right)\left|s_{1}\right|=-\sqrt{2} \eta_{1} V_{1}^{(1 / 2)}$, with $\eta_{1}=k_{1}-\delta_{31}>0$. Similarly, $\dot{V}_{2}=-\left(k_{2}-\delta_{32}\right)\left|s_{2}\right|=$ $-\sqrt{2} \eta_{2} V_{2}^{(1 / 2)}$, with $\eta_{2}=k_{2}-\delta_{32}>0$. From lemma and according to the finite-time Lyapunov stability criterion [29], the top and bottom Peltier control systems can reach $s_{1}=0$ and $s_{2}=0$ in finite time $t_{r 1}$ and $t_{r 2}$, respectively. Choosing $T=\max \left\{t_{f 1}, t_{f 2}, t_{r 1}, t_{r 2}\right\}$ and $t \geq T$, there is

$$
\left\{\begin{array}{l}
\dot{e}_{1}+\eta_{1} \operatorname{sign}\left(e_{1}\right)\left|e_{1}\right|^{\gamma_{1}}=0, \\
\dot{e}_{2}+\eta_{2} \operatorname{sign}\left(e_{2}\right)\left|e_{2}\right|^{\gamma_{2}}=0 .
\end{array}\right.
$$

(3) Proving that the state error of the system on the sliding mode surface can converge to the equilibrium point in finite time.

According to equation (16), the time to reach the equilibrium point can be calculated as follows on the sliding mode surface, respectively:

$$
\left\{\begin{array}{l}
t_{s 1}=\frac{\left|e_{1}\left(t_{r 1}\right)\right|^{1-\gamma_{1}}}{\eta_{1}\left(1-\gamma_{1}\right)}, \\
t_{s 2}=\frac{\left|e_{2}\left(t_{r 2}\right)\right|^{1-\gamma_{2}}}{\eta_{2}\left(1-\gamma_{2}\right)} .
\end{array}\right.
$$

It shows that on the terminal sliding mode surface (7), $e_{1}$ and $e_{2}$ can reach the equilibrium point in finite time. Therefore, $x_{1}$ approaches $x_{1 \text { ref }}$ in finite time and $x_{2}$ approaches $x_{2 \text { ref }}$ in finite time.

Remark. In the actual control system, since $\dot{e}_{i}$ in the sliding mode function $s_{i}(t)(i=1,2)$ contains the disturbance $d_{i}$, it is not feasible or easy to calculate $\operatorname{sign}\left(s_{i}\right)$ by calculating $s_{i}$. For calculating $\operatorname{sign}\left(s_{i}\right)$, a new function $g_{i}(t)$ is defined as follows:

$$
g_{i}(t)=\int_{0}^{t} s_{i}(t) \mathrm{d} t=e_{i}+\eta_{i} \int_{o}^{t} \operatorname{sign}\left(e_{i}\right)\left|e_{i}\right|^{\gamma_{i}},
$$

then $\operatorname{sign}\left(s_{i}\right)$ can be obtained as

$$
\operatorname{sign}\left(s_{i}\right)=\operatorname{sign}\left(g_{i}(t)-g_{i}(t-\Delta)\right),
$$

where $\Delta$ is the sampling time. Since $s_{i}(t)=$ $\lim _{t \rightarrow 0}\left(g_{i}(t)-g_{i}(t-\Delta)\right) / \Delta$, sampling time $\Delta$ can be choosed to judge the symbol of $s_{i}(t)$ according to the increase or decrease of $g_{i}(t)$ in the sampling time $\Delta$ [30].

\section{Simulation and Experiment}

In this section, simulations and experiments are carried out to verify the effectiveness of the proposed continuous integral terminal sliding mode controller for the double-layer Peltier system. To demonstrate the superiority of the proposed control strategy, a nonsmooth finite-time control strategy is employed for comparison. Nonsmooth control is a nonlinear control method between smooth control and discontinuous control. In our previous study of Peltier [6], under the premise that the disturbance can be accurately compensated, a finite-time nonsmooth feedback control strategy based on disturbance feedforward compensation was used. Utilizing the fractional power, the nonsmooth control allows the controller near the equilibrium point to have a larger control input, resulting in faster convergence of the system.

4.1. Simulation. In order to ensure the fairness of the comparison, the finite-time disturbance observer-based continuous integral terminal sliding mode controller is adopted for two Peltier structures (double-layer form and single-layer form). Also, two control systems choose the same value of parameters, as shown in Table 1 [6]. Setting the reference temperature to be $280 \mathrm{~K}$, when $t=10 \mathrm{~s}$, the disturbance starts to work and lasts for 5 seconds. The disturbance process is represented by a polynomial $\mathrm{d}(t)=-0.104 t^{3}+4.9536 t^{2}-78.786 t+418.23$. Figure 3 shows a curve of the disturbance $d_{1}$, the estimation $\widehat{d}_{1}$ of the disturbance, and the estimation error $\widetilde{d}_{1}$. About 0.2 second after the disturbance, the estimation error of disturbance converges to zero, which fully shows that the sliding mode observer 3 with finite-time convergence can accurately and quickly estimate the polynomial disturbance. The control response curves and the control input curves of the single-layer Peltier system and double-layer Peltier system are illustrated in Figures 4 and 5, respectively.

It can be found in Figure 4 that for the $20 \mathrm{~K}$ temperature drop, double-layer Peltier has stronger disturbance rejection and faster convergence than single-layer Peltier. In Figure 5, the input current of double-layer Peltier is smaller than that of single-layer Peltier. This results from that the double-layer Peltier divides the cooling temperature into two parts, one for the top layer and the other for the bottom layer. The top and bottom layers are cooled separately; therefore, each layer finishes a smaller temperature drop. Also, the smaller the temperature drop, the less energy needed, namely, the less control input required. In addition, when the temperature shows a large drop, the convergence speed of single-layer Peltier is limited due to the given control input amplitude. Therefore, double-layer Peltier can be adopted when larger cooling temperature difference and better control performance are needed.

Our previous work [6] has studied the nonsmooth finitetime control strategy of the double-layer Peltier system which is used as a comparison in this section. Under the condition of finite-time convergence, the advantages of the terminal sliding mode control (TSMC) over finite-time composite control (FTCC) are discussed here. The parameters of two controllers are listed in Table 2.

Figures 6 and 7 show the response curves and corresponding control input curves of FTCC and TSMC algorithms, respectively. From Figures 6 and 7, two algorithms have the same control input when the simulation starts. Also, near the equilibrium point, TSMC has faster 
Table 1: Control parameters of two Peltier control systems.

\begin{tabular}{lcc}
\hline Control parameters & Single-layer Peltier & Double-layer Peltier \\
\hline & $k=20, x_{\text {ref }}=280 K, \gamma=3 / 5$, & $k_{1}=20, k_{2}=20, x_{1 \mathrm{ref}}=280 K$, \\
Controller parameters & $\lambda=30, T_{o}=300 K, u_{\max }=5 A$, & $x_{2 \mathrm{ref}}=288 K, \eta_{1}=30, \eta_{2}=30$, \\
& $u_{o}=300 K, \gamma=3 / 5, u_{\max }=5 A$, & $u_{\min }=-5 A$
\end{tabular}

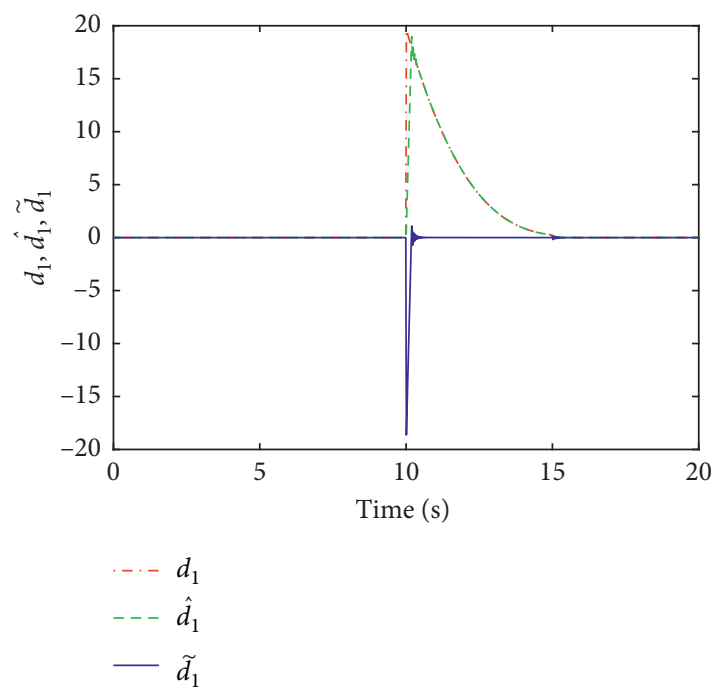

FIgURE 3: Curve of disturbance, estimation, and the estimation error.

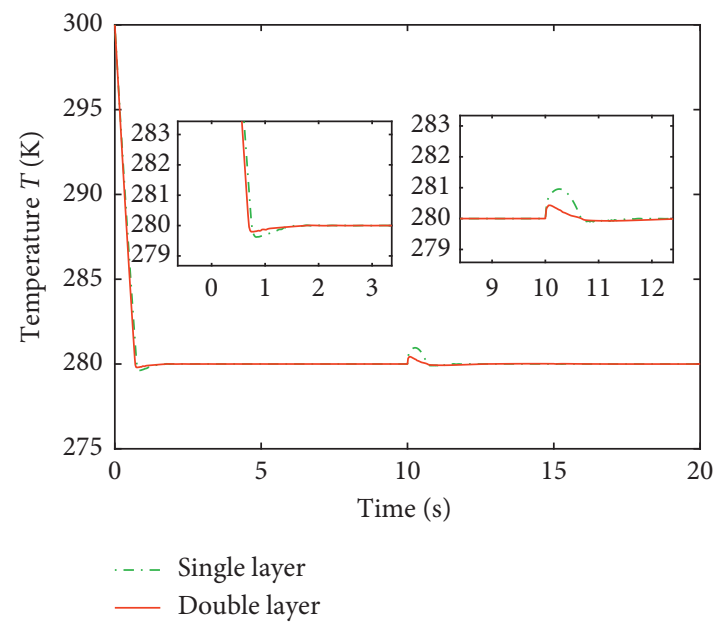

Figure 4: Control response curves of two Peltier control systems (simulation).

convergence than FTCC. When the disturbance acts on the system at $t=10 \mathrm{~s}$, TSMC needs $0.25 \mathrm{~s}$ to regain the balance, while FTCC requires $1.3 \mathrm{~s}$ to reach the set temperature again. These fully reveal the fast convergence and strong disturbance rejection of the TSMC proposed above. Moreover, it is observed from Figure 7 that the continuous integral sliding

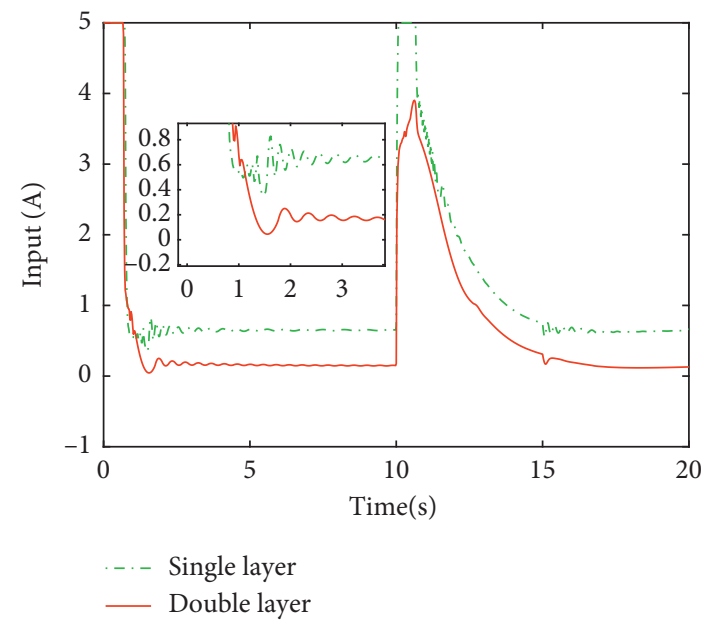

Figure 5: Control input curves of two Peltier control systems (simulation).

mode makes the control input of the system continuous and attenuates the chattering effectively.

4.2. Experiment. In order to apply the theory to practice, we designed a hardware platform [6] to compare two algorithms above, namely, FTCC and TSMC. Figure 8 shows the integrated design of the experimental hardware platform, and Figure 9 shows the actual equipment diagram of the experimental hardware platform. The following data would be obtained from the equipment diagram in Figure 9. This device takes a dual-core chip OAMP-1138 from TI company as the main controller, which is composed of ARM and DSP. ARM is responsible for nonreal-time tasks, including human-computer interaction, reading data measured by sensors, and sending data to the DSP. DSP is responsible for high-intensity data real-time processing and calculation, which means cyclically receiving data transmitted from the ARM for calculation processing.

The experiment was conducted at a room temperature of $23^{\circ} \mathrm{C}$ (about $300 \mathrm{~K}$ ). Before the experiment, the surface temperature of Peltier was kept at room temperature. Fingers were washed and wrapped in warm towels (about $45^{\circ} \mathrm{C}$ ). Once the experiment started, the finger was placed on the Peltier chip for 10 seconds and removed after another 5 seconds. During this contact interval, the surface temperature became stable again rapidly. The surface temperature data are collected based on FTCC and TSMC algorithms, respectively, and illustrated in Figure 10. 
TABLE 2: Control parameters of FTCC and TSMC.

\begin{tabular}{lcc}
\hline Control parameters & FTCC & TSMC \\
\hline & $x_{1 \mathrm{ref}}=280 K, x_{2 \mathrm{ref}}=288 K$, & $k_{1}=20, k_{2}=20, x_{1 \mathrm{ref}}=280 K$, \\
Controller parameters & $T_{o}=300 K, \alpha=3 / 5, u_{\max }=5 A$, & $x_{2 \mathrm{ref}}=288 K, \eta_{1}=30, \eta_{2}=30$, \\
& $T_{o}=300 K, \gamma=3 / 5, u_{\max }=5 A$, & $u_{\min }=-5 A$ \\
& & $k_{11}=15, k_{12}=15, k_{21}=10$, \\
Observer parameters & $\lambda_{1}=10, \lambda_{2}=5, \lambda_{3}=10$, & $k_{22}=10, k_{31}=3, k_{32}=3, \tau=-0.2$ \\
\hline
\end{tabular}

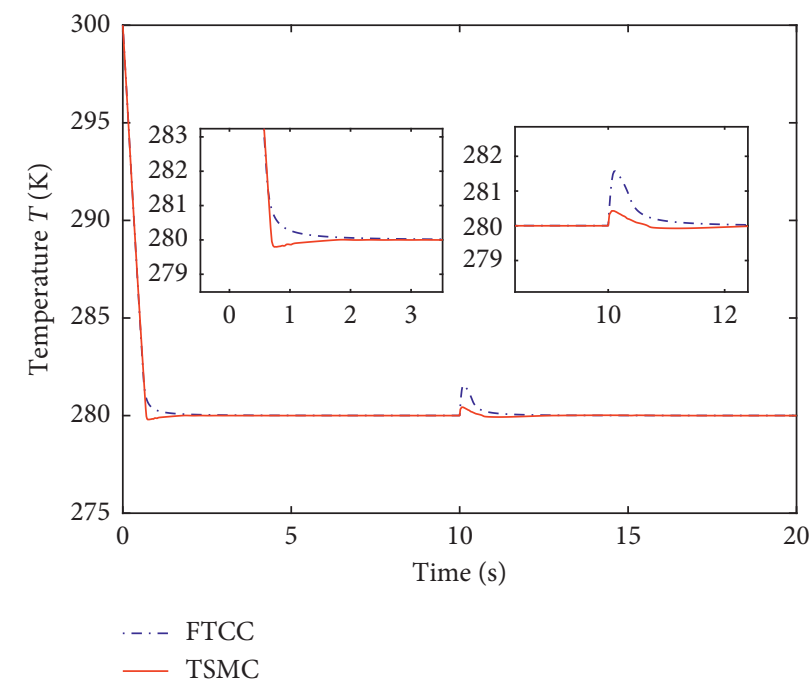

FIGURE 6: Control response curves of FTCC and TSMC (simulation).

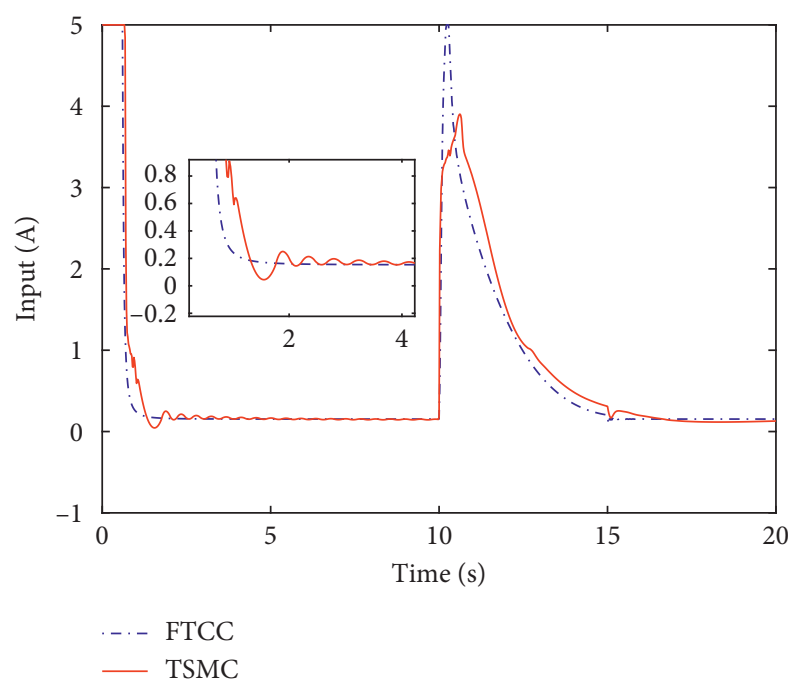

FIgURE 7: Control input curves of FTCC and TSMC (simulation).

The experimental temperature response curves and control input curves of FTCC and TSMC are shown in Figures 10 and 11, which are similar to the simulation curves. Although the TSMC strategy is slightly overshot, the sliding mode control with strong disturbance rejection has a better performance than the nonsmooth feedback control. From Figure 11, the control input of the two algorithms is almost equal when the input becomes stable. Then about $t=11 \mathrm{~s}$, the double-layer Peltier overcomes the thermal flow disturbance of fingers and becomes stable again under the FTCC and TSMC algorithms. The experimental control input curves and the simulation control input curves are somewhat different because the finger is not a heat source for continuous excitation during the experiment. During the 


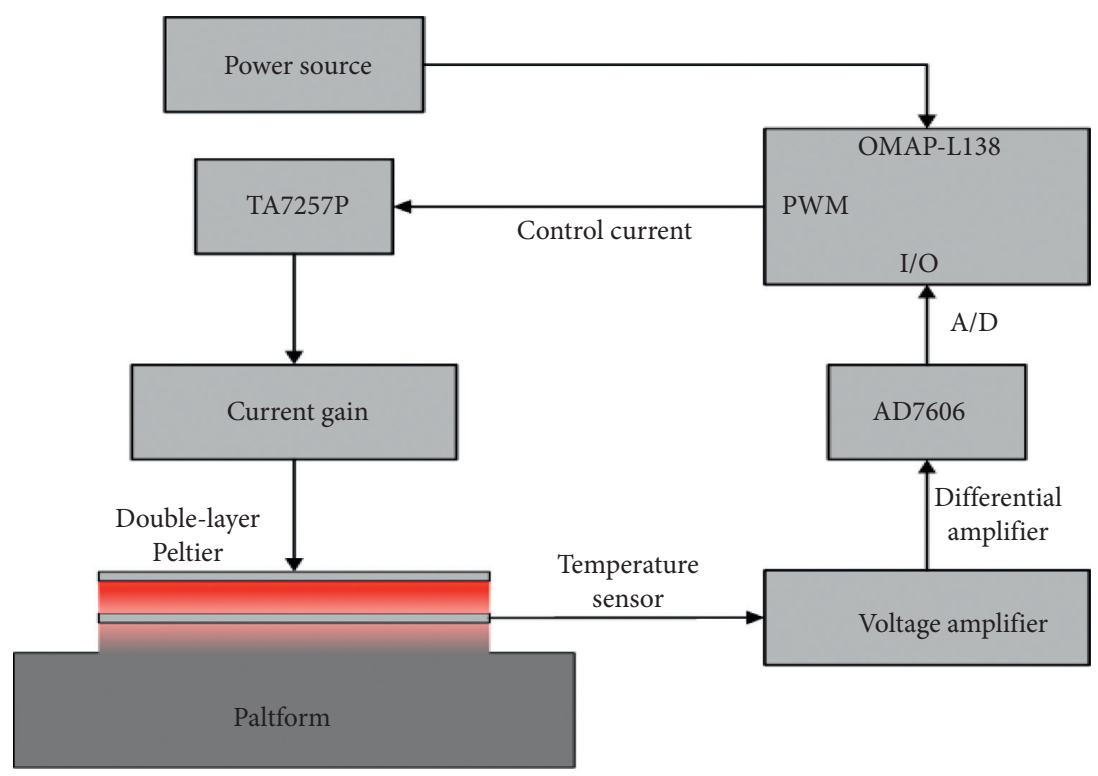

FIGURE 8: Integrated design of the experimental hardware platform.

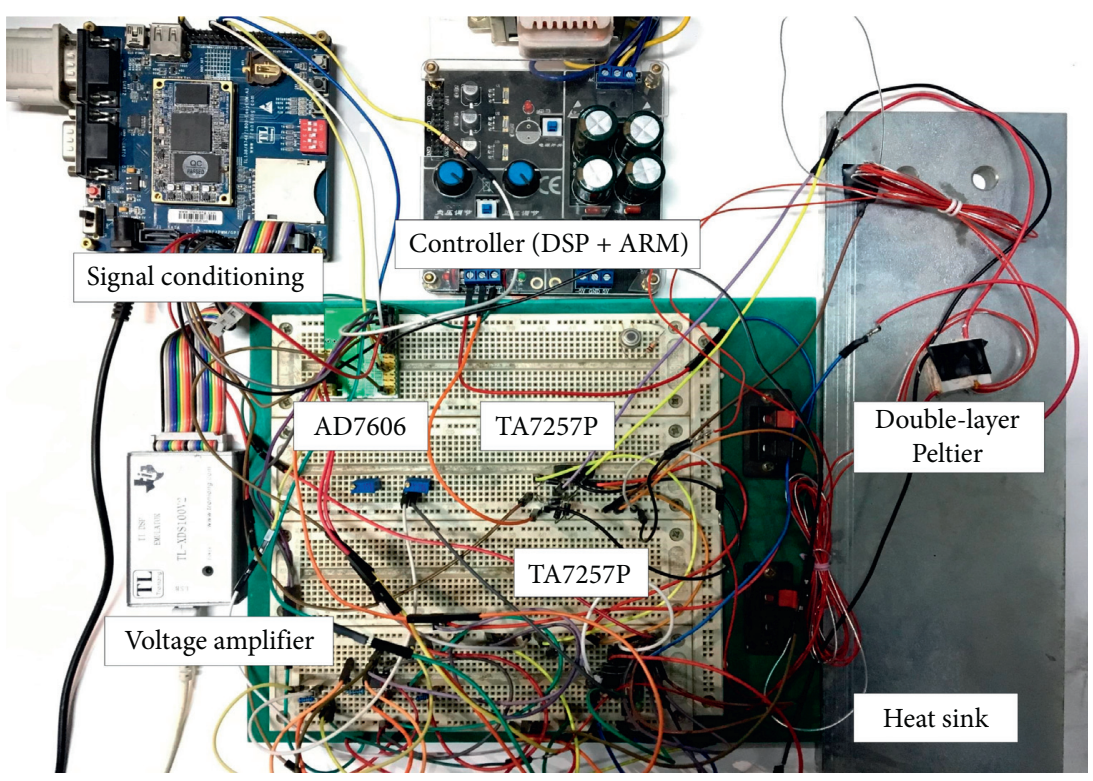

FIGURE 9: Equipment diagram of the experimental hardware platform.

heat exchange, when about $t=11 \mathrm{~s}$, the temperature of the finger was very close to the temperature of the Peltier. Then, Peltier would be in a stable state, and the finger did not disturb the Peltier surface temperature.

Table 3 lists the performance indexes of the single-layer TSMC algorithm (STSMC), double-layer TSMC algorithm (DTSMC), and double-layer FTCC algorithm (DFTCC). TDR stands for temperature drop rate. Overshoot tolerance range is chosen to be $2 \%$. ST represents stable time. MPTR stands for maximum percent temperature rise which is the maximum value of the temperature rise after disturbance is acted. As can be seen from Table 3, the double-layer Peltier has a faster temperature drop rate and less stable time than the single-layer Peltier. Since both DTSMC and DFTCC converge in finite time, DFTCC and DTSMC have a similar temperature drop rate. Because the sliding mode control system is robust, STSMC and DTSMC have smaller maximum temperature rise percentage than DFTCC. Compared to the nonsmooth finite-time control, the sliding mode control performs better in moving the system state to the set 


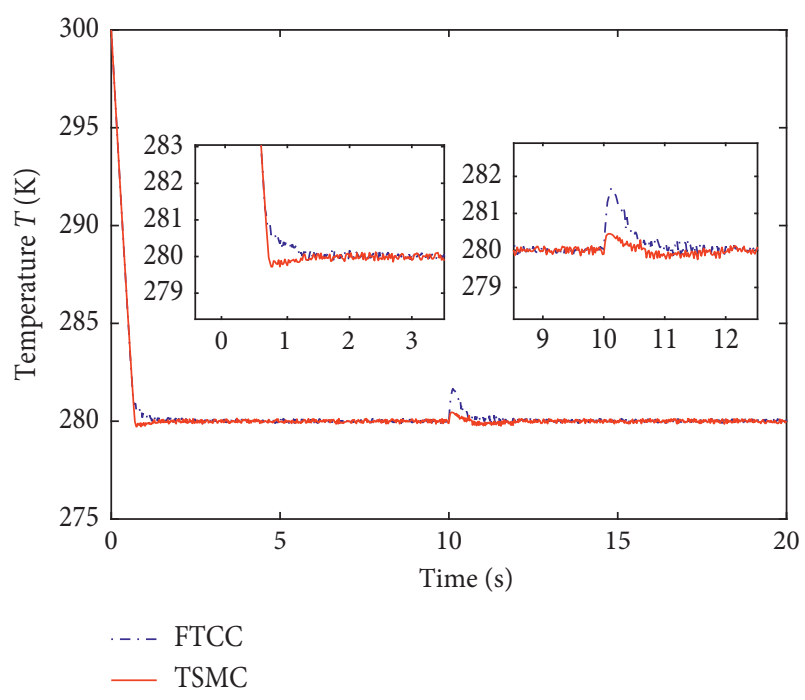

Figure 10: Control response curves of FTCC and TSMC (experiment).

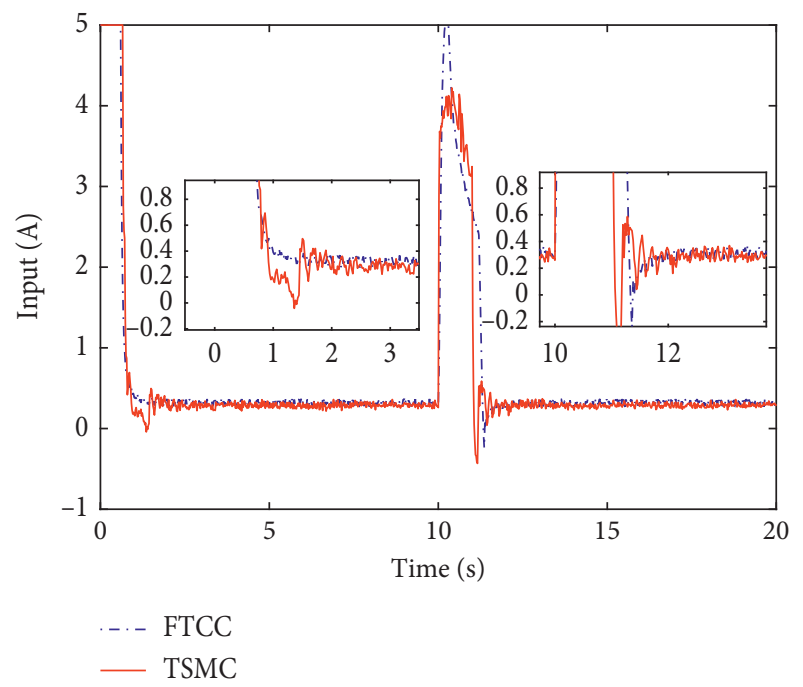

FIGURE 11: Control input curves of FTCC and TSMC (experiment).

TABle 3: Control parameters of the Peltier system.

\begin{tabular}{lccc}
\hline & STSMC & DTSMC & DFTCC \\
\hline TDR $\left({ }^{\circ} \mathrm{C} / \mathrm{s}\right)$ & 24.26 & 27.30 & 28.48 \\
ST $(\mathrm{s})$ & 0.57 & 0.14 & 0.53 \\
MPTR (\%) & 4.80 & 2.15 & 7.80 \\
\hline
\end{tabular}

sliding mode state. The sliding mode is artificially designed. Also, it makes the sliding mode control robust to perturbation of those parameters and unmodeled dynamics. Also, for finite-time convergence, the sliding mode control can suppress these disturbances and the influence of unknown factors. Therefore, the sliding mode control has a stronger disturbance rejection performance.

\section{Conclusion}

In this paper, the problem of the finite-time tracking control of the surface temperature of the double-layer Peltier system has been addressed with the help of the continuous integral terminal sliding mode control strategy based on the finitetime disturbance observer. Firstly, the model of the doublelayer Peltier system has been formulated well via the thermal network model. Secondly, the finite-time disturbance observer based on the system model has been adopted to estimate the external thermal flow disturbances. Then, the compound continuous integral terminal sliding mode control strategy has been developed and the continuous control input has reduced the sliding mode chattering obviously. Finally, compared with the single-layer Peltier, the advantages of double-layer Peltier in larger temperature drop control performance have been verified. Besides, compared with the double-layer Peltier nonsmooth finite-time control, it has been shown that the continuous integral terminal sliding mode control for double-layer Peltier system has a stronger disturbance rejection performance. It is worth mentioning that the impedance of the sensor itself is neglected in the experiment, which should be taken into account for accurate modeling. Moreover, the Thomson effect [31] will affect the accuracy of the control in the application where the refrigeration temperature difference is larger. The precise estimation of the disturbance and the improvement of the control accuracy will be further studied in the future.

\section{Data Availability}

No additional data were used to support this study.

\section{Conflicts of Interest}

The authors declare that they have no conflicts of interest.

\section{References}

[1] C. Chen, Y. Wang, and S. Li, "Generalized proportional integral observer-based composite control method for robotic thermal tactile sensor with disturbances," International Journal of Advanced Robotic Systems, vol. 14, no. 3, Article ID 1729881417710033, 2017.

[2] T. Metzger and R. P. Huebener, "Modelling and cooling behaviour of Peltier cascades," Cryogenics, vol. 39, no. 3, pp. 235-239, 1999.

[3] R. T. Delves, "The prospects for Ettingshausen and Peltier cooling at low temperatures," British Journal of Applied Physics, vol. 13, no. 9, pp. 440-445, 1962.

[4] S. Sastry, Nonlinear Systems: Analysis, Stability, and Control, Vol. 10, Springer Science \& Business Media, Berlin, Germany, 2013.

[5] M. Deng, S. Wen, and A. Inoue, "Operator-based robust nonlinear control for a Peltier actuated process," Measurement and Control, vol. 44, no. 4, pp. 116-120, 2013.

[6] C. Chen, S. Ding, and S. Li, "Finite-time control for doublelayer Peltier system based on finite-time observer," Advances in Mechanical Engineering, vol. 11, no. 3, Article ID $1687814019836852,2019$. 
[7] G. Wen, Z. Duan, Y. Zhao, W. Yu, and J. Cao, "Robust containment tracking of uncertain linear multi-agent systems: a non-smooth control approach," International Journal of Control, vol. 87, no. 12, pp. 2522-2534, 2014.

[8] Y. Shtessel, C. Edwards, L. Fridman, and A. Levant, "Higherorder sliding mode controllers and differentiators," Sliding Mode Control and Observation, vol. 89, no. 9, pp. 213-249, 2014.

[9] M. Bouri and D. Thomasset, "Sliding control of an electropneumatic actuator using an integral switching surface," IEEE Transactions on Control Systems Technology, vol. 9, no. 2, pp. 368-375, 2001.

[10] V. Utkin, J. Guldner, and J. Shi, Sliding Mode Control in Electro-Mechanical Systems, CRC Press, Boca Raton, FL, USA, 2009.

[11] B. Yang, T. Yu, H. Shu, J. Dong, and L. Jiang, "Robust slidingmode control of wind energy conversion systems for optimal power extraction via nonlinear perturbation observers," Applied Energ, vol. 210, pp. 711-723, 2018.

[12] İ. Eker, "Sliding mode control with PID sliding surface and experimental application to an electromechanical plant," ISA Transactions, vol. 45, no. 1, pp. 109-118, 2006.

[13] V. Utkin and H. Lee, "Chattering problem in sliding mode control systems," Analysis and Design of Hybrid Systems 2006, vol. 39, no. 5, p. 1, 2006.

[14] A. Levant, "Sliding order and sliding accuracy in sliding mode control," International Journal of Control, vol. 58, no. 6, pp. 1247-1263, 1993.

[15] G. Bartolini, A. Ferrara, and E. Usai, "Chattering avoidance by second-order sliding mode control," IEEE Transactions on Automatic Control, vol. 43, no. 2, pp. 241-246, 2002.

[16] V. Nekoukar and A. Erfanian, "Adaptive fuzzy terminal sliding mode control for a class of MIMO uncertain nonlinear systems," Fuzzy Sets and Systems, vol. 179, no. 1, pp. 34-49, 2011.

[17] J. Lian and J. Zhao, "Robust H-infinity integral sliding mode control for a class of uncertain switched nonlinear systems," Journal of Control Theory and Applications, vol. 8, no. 4, pp. 521-526, 2010.

[18] I. C. Baik, K. H. Kim, and M. J. Youn, "Robust nonlinear speed control of PM synchronous motor using boundary layer integral sliding mode control technique," IEEE Transactions on Control Systems Technology, vol. 8, no. 1, pp. 47-54, 2000.

[19] H. Jahanshahi, A. Yousefpour, Z. Wei, R. Alcaraz, and S. Bekiros, "A financial hyperchaotic system with coexisting attractors: dynamic investigation, entropy analysis, control and synchronization," Chaos, Solitons \& Fractals, vol. 126, pp. $66-77,2019$.

[20] H. Jahanshahi, A. Yousefpour, J. M. Munoz-Pacheco, I. Moroz, Z. Wei, and O. Castillo, "A new multi-stable fractional-order four-dimensional system with self-excited and hidden chaotic attractors: dynamic analysis and adaptive synchronization using a novel fuzzy adaptive sliding mode control method," Applied Soft Computing, vol. 87, Article ID 105943, 2020.

[21] A. Yousefpour and H. Jahanshahi, "Fast disturbance-observer-based robust integral terminal sliding mode control of a hyperchaotic memristor oscillator," The European Physical Journal Special Topics, vol. 228, no. 10, pp. 2247-2268, 2019.

[22] S. Li, M. Zhou, and X. Yu, "Design and implementation of terminal sliding mode control method for PMSM speed regulation system," IEEE Transactions on Industrial Informatics, vol. 9, no. 4, pp. 1879-1891, 2013.
[23] H. Morimitsu and S. Katsura, "Two-Degree-of-Freedom robust temperature control of peltier device based on heat disturbance observer," Electrical Engineering in Japan, vol. 184, no. 1, pp. 66-74, 2013.

[24] Y. Feng, X. Yu, and Z. Man, "Non-singular terminal sliding mode control of rigid manipulators," Automatica, vol. 38, no. 12, pp. 2159-2167, 2002.

[25] H. Du, C. Qian, S. Yang, and S. Li, "Recursive design of finitetime convergent observers for a class of time-varying nonlinear systems," Automatica, vol. 49, no. 2, pp. 601-609, 2013.

[26] V. I. Utkin, "Sliding modes in control and optimization," Communications and Control Engineering, vol. 189, no. 3, pp. 1372-1379, 1992.

[27] S. P. Bhat and D. S. Bernstein, "Finite-time stability of continuous autonomous systems," SIAM Journal on Control and Optimization, vol. 38, no. 3, pp. 751-766, 2000.

[28] S. Li and Y.-P. Tian, "Finite-time stability of cascaded timevarying systems," International Journal of Control, vol. 80, no. 4, pp. 646-657, 2007.

[29] S. Li and Y.-P. Tian, "Finite-time Lyapunov stability analysis and its application to atmospheric predictability," Journal of the Atmospheric Sciences, vol. 50, no. 11, pp. 1531-1543, 1993.

[30] Y. Feng, F. Han, and X. Yu, "Chattering free full-order slidingmode control," Automatica, vol. 50, no. 4, pp. 1310-1314, 2014.

[31] R. R. Heikes and R. W. Ure, "Thermoelectricity: science and engineering," Journal of Chemical Education, vol. 39, no. 5, p. $274,1960$. 